\title{
Design and Analysis of a Sensing Error-Aware MAC Protocol for Cognitive Radio Networks
}

\author{
Donglin $\mathrm{Hu}$ and Shiwen Mao \\ Department of Electrical and Computer Engineering, Auburn University, Auburn, AL \\ Email: dzh0003@auburn.edu, smao@ieee.org
}

\begin{abstract}
In this paper, we present a spectrum sensing erroraware MAC protocol for a cognitive radio (CR) network collocated with multiple primary networks. We explicitly consider sensing errors in the CR MAC design, since such errors are inevitable for practical spectrum sensors. Two spectrum sensing polices are presented with which secondary users collaboratively sense the licensed channels. The sensing policies are then incorporated into $p$-Persistent CSMA to coordinate dynamic spectrum access for CR network users. We present an analysis of the interference and throughput performance of the proposed $C R$ MAC, and find the analysis highly accurate in our simulation studies. The proposed sensing error-aware CR MAC protocol outperforms two existing approaches with considerable margins in our simulations, which justify the importance of considering spectrum sensing errors in CR MAC design.
\end{abstract}

\section{INTRODUCTION}

A cognitive radio $(\mathrm{CR})$ is a frequency-agile wireless communication device with intelligent decision-making and a monitoring interface that enables dynamic spectrum access [1]. The CR concept represents a significant paradigm change in spectrum regulation and utilization, i.e., from exclusive use of spectrum by licensed users (or, primary users) to dynamic spectrum access for unlicensed users (or, secondary users). The high potential of CRs has attracted considerable efforts from the wireless community recently for more efficient spectrum management policies and techniques [1], [2].

Although the basic concept of $\mathrm{CR}$ is intuitive, it is challenging to design efficient cognitive network protocols to fully capitalize CR's potential. In order to exploit transmission opportunities in licensed bands, the tension between primary user protection and secondary user spectrum access should be judiciously balanced. Important design factors include (i) how to identify transmission opportunities, (ii) how secondary users determine, among the licensed channels, which channel(s) and when to access for data transmission, and (iii) how to avoid harmful interference to primary users under the omnipresent of spectrum (or, channel) sensing errors. These are the problems that should be addressed in the medium access control (MAC) protocol design for CR networks. Although good understandings on licensed channel processes have been gained recently [3], [4], there is still a critical need to develop analytical models that take channel sensing errors into account for guiding the design of CR MAC protocols.

In this paper, we present a channel sensing error-aware MAC protocol for a CR network collocated with $M$ primary networks. We assume primary users access the licensed chan- nels following a synchronous time slot structure [1], [5]. The channel states are independent to each other and each evolves over time following a discrete-time Markov process [1], [3]. Secondary users use their software-defined radio (SDR)-based transceivers to tune to any of the $M$ licensed channels, to sense and estimate channel status and to access the channels when they are found (or, believed) to be available.

We explicitly consider channel sensing errors in the design of the CR MAC protocol. It has been shown in prior work that generally there are two kinds of channel sensing errors: with a false alarm, a spectrum opportunity will be wasted, while a miss detection may lead to collision with primary users. We consider both sensing errors in our CR MAC design, which are unavoidable for practical spectrum sensors [1].

Specifically, we develop two channel sensing polices where secondary users collaboratively sense the licensed channels. With the memoryless sensing policy, each secondary user chooses one of the $M$ licensed channels to sense (and to access after a successful sensing phase) with equal probability. During the sensing phase, secondary users also exchange sensing results through a separate control channel. This sensing policy is further improved with a mechanism to spread out secondary users to sense different channels, therefore reducing the chance that a channel is not sensed by any of the users, and by considering the autocorrelation of channel processes to obtain more accurate sensing results. This is termed improved sensing policy. These two sensing polices are then incorporated into the $p$-Persistent Carrier Sense Multiple Access (CSMA) mechanism to make a sensing error-aware CR MAC protocol. We analyze the proposed CR MAC protocol with respect to its interference and throughput performance with closed-form expressions. Primary user protection is achieved via tunning the channel access probability $p$ of $p$-Persistent CSMA according to the interference analysis. We find that the analysis is highly accurate as compared to simulation results. In addition, the proposed sensing error-aware CR MAC protocol outperforms two existing schemes with considerable gain margins, which justify the importance of considering channel sensing errors in CR MAC design.

The rest of this paper is organized as follows. We describe the network model and assumptions in Section II. We then present the proposed CR MAC protocol and analyze its performance in Section III. Our simulation studies are provided in Section IV. Section V discusses related work and Section VI concludes this paper. 


\section{Network Model ANd Assumptions}

Consider $M$ primary networks, each allocated with a licensed channel. We assume the primary users access the channels following a synchronous slot structure. The channel states are independent to each other and each of the $M$ channels evolves over time following a discrete-time two-state Markov process [1], [3], [5], [6]. We define the network state vector in slot $t$ as $\vec{S}(t)=\left[S_{1}(t), S_{2}(t), \ldots, S_{M}(t)\right]$, where $S_{m}(t)$ denotes the state of channel $m$ with idle $\left(S_{m}(t)=0\right)$ and busy $\left(S_{m}(t)=1\right)$ states, for $m=1,2, \cdots, M$.

Let $\lambda_{m}$ and $\mu_{m}$ be the transition probability of remaining in state 0 and that from 1 to 0 for channel $m$, respectively. Let $\eta_{m}=\operatorname{Pr}\left(S_{m}=1\right)$ denote the utilization of the $m$-th channel with respect to primary user transmissions. We have

$$
\eta_{m}=\lim _{T \rightarrow \infty} \frac{1}{T} \sum_{t=1}^{T} S_{m}(t)=\frac{1-\lambda_{m}}{1-\lambda_{m}+\mu_{m}} .
$$

Let $\zeta_{m}=\operatorname{Pr}\left(S_{m}=0\right)$ be the probability that channel $m$ is idle. We then have

$$
\zeta_{m}=1-\operatorname{Pr}\left(S_{m}=1\right)=\frac{\mu_{m}}{1-\lambda_{m}+\mu_{m}} .
$$

We assume a secondary network collocated with the $M$ primary networks, within which there are $N$ secondary users that take advantage of dynamic spectrum access for data transmission over the $M$ channels. For protection of primary users on channel $m$, the probability of collision caused by secondary user transmissions to primary users should be upper bounded by a prescribed threshold $\gamma_{m}$, for $m=1,2, \cdots, M$.

As in prior work [3], [5], [7], we assume that each secondary user is equipped with two transceivers: a control transceiver that operates over a dedicated control channel, which we assume is always available (i.e., a channel in the industrial, scientific and medical (ISM) band), and a data transceiver that is used to for data communication through the $M$ channels. Secondary users also use their transceivers to obtain information of available channels. The data transceiver consists of an SDR that can be tuned to any of the $M$ licensed channels to transmit and receive data from there.

\section{SENSING ERror-AwARE CR MAC Protocol}

For the CR network described above, we develop a MAC protocol for dynamic spectrum access that considers sensing errors. The time slot structure of the proposed MAC is shown in Fig. 1, which consists of a sensing phase and a transmission phase. The sensing phase is further divided into $\bar{K}$ minislots, within which each secondary user picks a channel to sense. CR users access the channels for data transmission during the transmission phase. We first discuss the two key components of the proposed protocol, i.e., channel sensing and channel access, and then analyze its performance with respect to primary user protection and achievable throughput.

\section{A. Sensing Phase}

The first key element of the proposed MAC protocol is channel sensing. Although precise and timely channel state information is highly desirable for dynamic spectrum access

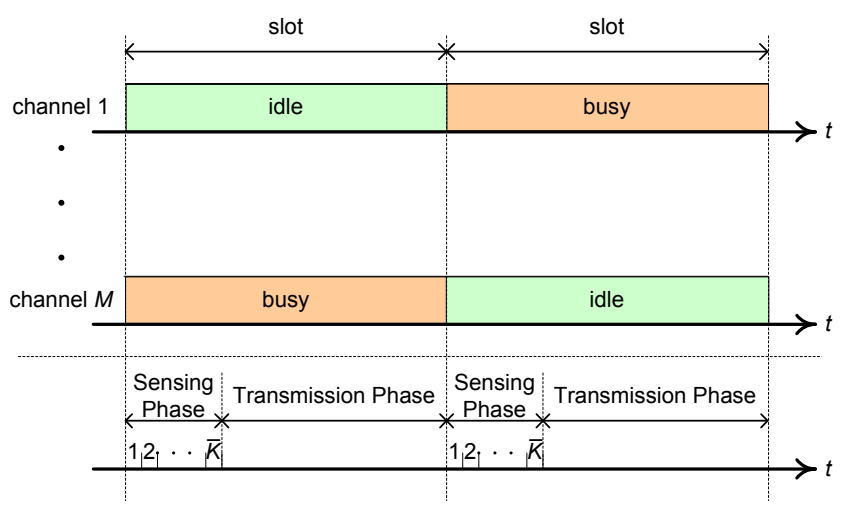

Fig. 1. The time slot structure of the proposed MAC protocol.

and primary user protection, contiguous full-spectrum sensing is both energy inefficient and hardware demanding. Since we assume a secondary user is equipped with one antenna for spectrum sensing, i.e., the data transceiver with SDR capability, only one of the channels can be sensed at a time.

During the sensing phase (see Fig. 1), a secondary user picks a licensed channel and keeps on sensing it for one or multiple mini-slots. As discussed, two kinds of detection errors may occur: false alarm and miss detection. We assume all secondary users have the same probability of detection errors over channel $m, m=1,2, \cdots, M$. Let $\epsilon_{m}$ and $\delta_{m}$ denote the probabilities of false alarm and miss detection on channel $m$, respectively. The spectrum sensing performance can be represented by the Receiver Operation Characteristic (ROC) curve, which plots $1-\delta_{m}$ as a function of $\epsilon_{m}$ [1]. For a specific channel $m$ in a certain time slot $t$, the sensing error probabilities can be written as:

$$
\begin{cases}\operatorname{Pr}\left(W_{m, i}=1 \mid S_{m}=0\right)=\epsilon_{m}, & \forall i=1,2, \cdots \\ \operatorname{Pr}\left(W_{m, i}=0 \mid S_{m}=1\right)=\delta_{m}, & \forall i=1,2, \cdots,\end{cases}
$$

where $W_{m, i}$ is the $i$ th sensing result of channel $m$ and $S_{m}$ is state of channel $m$.

We assume that the sensing results from different users are independent and the sensing results in different mini-slots are also independent to each other. The conditional probability that channel $m$ is available after the $k$ th sensing mini-slot, denoted by $a_{m, k}$, can be derived as

$$
\begin{aligned}
& a_{m, k}=\operatorname{Pr}\left(S_{m}=0 \mid W_{m, 1}=\theta_{m, 1}, \cdots, W_{m, k}=\theta_{m, k}\right) \\
& =\frac{\operatorname{Pr}\left(W_{m, i}=\theta_{m, i}, i=1, \cdots, k \mid S_{m}=0\right) \operatorname{Pr}\left(S_{m}=0\right)}{\sum_{j=0}^{1} \operatorname{Pr}\left(W_{m, i}=\theta_{m, i}, i=1, \cdots, k \mid S_{m}=j\right) \operatorname{Pr}\left(S_{m}=j\right)} \\
& =\left[1+\alpha_{m}^{d_{m}} \beta_{m}^{k-d_{m}} \frac{\operatorname{Pr}\left(S_{m}=1\right)}{\operatorname{Pr}\left(S_{m}=0\right)}\right]^{-1}=\left(1+\alpha_{m}^{d_{m}} \beta_{m}^{k-d_{m}} \frac{\eta_{m}}{\zeta_{m}}\right)^{-1}
\end{aligned}
$$

where $d_{m}$ is the number of observations that the sensing result on channel $m$ is 0 , and $\alpha_{m}$ and $\beta_{m}$ are defined as follows.

$$
\begin{gathered}
\alpha_{m}=\frac{\operatorname{Pr}\left(W_{m, i}=0 \mid S_{m}=1\right)}{\operatorname{Pr}\left(W_{m, i}=0 \mid S_{m}=0\right)}=\frac{\delta_{m}}{1-\epsilon_{m}}, \text { for } \theta_{m, i}=0 \\
\beta_{m}=\frac{\operatorname{Pr}\left(W_{m, i}=1 \mid S_{m}=1\right)}{\operatorname{Pr}\left(W_{m, i}=1 \mid S_{m}=0\right)}=\frac{1-\delta_{m}}{\epsilon_{m}}, \text { for } \theta_{m, i}=1 .
\end{gathered}
$$


By abuse of notation, we also use $a_{m, k}$ to denote the conditional probability that channel $m$ is available when there are $k$ sensing results available. Therefore (4) is also for the case of one user that senses channel $m$ for $k$ mini-slots (i.e., there are $k * 1$ sensing samples available).

During the sensing phase, each secondary user chooses one channel to sense with equal probability at the beginning of the time slot. Secondary users also report their sensing results over the control channel, and share the corresponding channel information during the mini-slots. Two threshold probabilities are used. If the availability of channel $m$ is below $\Theta_{0}$, the channel is believed to be busy and the secondary users will wait till the next time slot to start sensing again. If the channel $m$ availability is between $\Theta_{0}$ and $\Theta_{1}$, secondary users will keep on sensing the same channel. If the channel $m$ availability exceeds $\Theta_{1}$, the channel is believed to be idle and the secondary users stop sensing and prepare to access the channel (see Section III-B). The stop time $K_{m}$ when secondary users stop sensing channel $m$, is a random variable that takes value between 1 and $\bar{K}$, the maximum number of mini-slots that can be used for sensing (see Fig. 1). Let $T_{s}, T_{m s}$, and $T_{\text {data }}$ be the duration of a time slot, a mini-slot, and the transmission phase, respectively (see Fig. 1), we have

$$
T_{s}=\bar{K} \times T_{m s}+T_{d a t a} .
$$

When there are $k$ sensing results available (equivalent to that one user senses channel $m$ for $k$ mini-slots), we define three sets of estimates for the channel $m$ state, as:

$$
\begin{aligned}
\Psi_{m, k}^{0} & =\left\{d_{m} \mid a_{m, k} \leq \Theta_{0}, \forall 0 \leq d_{m} \leq k\right\} \\
\Psi_{m, k}^{1} & =\left\{d_{m} \mid a_{m, k} \geq \Theta_{1}, \forall 0 \leq d_{m} \leq k\right\} \\
\Psi_{m, k}^{2} & =\left\{d_{m} \mid \Theta_{0}<a_{m, k}<\Theta_{1}, \forall 0 \leq d_{m} \leq k\right\} \\
& =\frac{\left(\Psi_{m, k}^{0} \cup \Psi_{m, k}^{1}\right)}{}
\end{aligned}
$$

where $d_{m}$ is the number of observations that the sensing result on channel $m$ is 0 . We then present two channel sensing policies based on this classification in the following.

1) Memoryless Sensing Policy: We propose a memoryless sensing policy in this section where secondary users cooperatively sense the licensed channels. We call it "memoryless" since it does not consider the sensing and analysis results in the previous time slot for simplicity. Specifically, each secondary user chooses one of the $M$ licensed channels to sense with equal probability. Channel selections of the $N$ secondary users are independent and identically distributed (i.i.d.).

Let $U_{m}$ be the random variable representing the number of secondary users that select channel $m$ to sense. The probability that $u_{m}$ users choose channel $m$ to sense is

$$
\operatorname{Pr}\left(U_{m}=u_{m}\right)=\left(\begin{array}{c}
N \\
u_{m}
\end{array}\right)\left(\frac{1}{M}\right)^{u_{m}}\left(\frac{M-1}{M}\right)^{N-u_{m}}
$$

The joint distribution that there are $u_{1}$ secondary users sensing channel $1, u_{2}$ secondary users sensing channel $2, \cdots$, and $u_{M}$ secondary users sensing channel $M$ is

$$
\begin{aligned}
& \operatorname{Pr}\left(u_{1}, u_{2}, \cdots, u_{M}\right) \\
= & \begin{cases}\prod_{m=1}^{M} \operatorname{Pr}\left(U_{m}=u_{m}\right), & \text { if } \sum_{m=1}^{M} u_{m}=N \\
0, & \text { otherwise. }\end{cases}
\end{aligned}
$$

We next derive the conditional probability that secondary users compete for channel after the sensing phase stops at mini-slot $K_{m}$. Note that the stop time is $K_{m}$ not only means that secondary users stop sensing channel $m$ after this minislot, but also indicates that the estimated availability of channel $m$ is already greater than the threshold $\Theta_{1}$, and thus these secondary users think channel $m$ is idle and are ready to access the channel for data transmission. There are $U_{m}$ users sensing channel $m$ and $U_{m} K_{m}$ observations available after mini-slot $K_{m}$, which is also a random variable. We first derive the conditional probability for $K_{m}=1$, as

$$
\begin{aligned}
& \operatorname{Pr}\left(K_{m}=1 \mid U_{m}=u, S_{m}=0\right)=\operatorname{Pr}\left(a_{m, u} \geq \Theta_{1}\right) \\
= & \sum_{d_{m}^{1} \in \Psi_{m, u}^{1}}\left(\begin{array}{r}
u \\
d_{m}^{1}
\end{array}\right)\left[\left(\epsilon_{m}\right)^{u-d_{m}^{1}}\left(1-\epsilon_{m}\right)^{d_{m}^{1}}\right],
\end{aligned}
$$

where $d_{m}^{1}$ is the number of observations that the sensing results are 0 in the first mini-slot.

Following similar reasoning as in (13), we can obtain the conditional probability that the stop time $K_{m}$ is 2 as

$$
\begin{aligned}
& \operatorname{Pr}\left(K_{m}=2 \mid U_{m}=u, S_{m}=0\right) \\
= & \operatorname{Pr}\left[\left(\Theta_{0}<a_{m, u}<\Theta_{1}\right) \cap\left(a_{m, 2 u} \geq \Theta_{1}\right)\right] \\
= & \sum_{D_{m}^{2} \in \Psi_{m, 2 u}^{1}} \sum_{d_{m}^{1} \in \Psi_{m, u}^{2}}\left(\begin{array}{c}
u \\
d_{m}^{1}
\end{array}\right)\left(\begin{array}{c}
u \\
d_{m}^{2}
\end{array}\right)\left[\left(\epsilon_{m}\right)^{2 u-D_{m}^{2}}\left(1-\epsilon_{m}\right)^{D_{m}^{2}}\right],
\end{aligned}
$$

where $\Psi_{m, k}^{2}$ is defined in (10) and $D_{m}^{2}=d_{m}^{1}+d_{m}^{2}$. We can derive the conditional probability that the stop time is $k$ as:

$$
\begin{aligned}
& \operatorname{Pr}\left(K_{m}=k \mid U_{m}=u, S_{m}=0\right) \\
= & \operatorname{Pr}\left[\left(\Theta_{0}<a_{m, u}<\Theta_{1}\right) \cap\left(\Theta_{0}<a_{m, 2 u}<\Theta_{1}\right) \cap\right. \\
= & \sum_{D_{m}^{k} \in \Psi_{m, k u}^{1}} \sum_{D_{m}^{k-1} \in \Psi_{m,(k-1) u}^{2}} \cdots \sum_{d_{m}^{1} \in \Psi_{m, u}^{2}} \\
& \left(\begin{array}{c}
u \\
d_{m}^{1}
\end{array}\right)\left(\begin{array}{c}
u \\
d_{m}^{2}
\end{array}\right) \cdots\left(\begin{array}{c}
u \\
d_{m}^{k}
\end{array}\right)\left[\left(\epsilon_{m}\right)^{k u-D_{m}^{k}}\left(1-\epsilon_{m}\right)^{D_{m}^{k}}\right],
\end{aligned}
$$

where $k=1, \cdots, \bar{K}$ and $D_{m}^{k}=\sum_{i=1}^{k} d_{m}^{i}$. We will apply these results in Section III-D to derive the throughput of the CR network by the law of total probability.

2) Improved Sensing Policy : Under the memoryless sensing policy, some channels may not be sensed with a sufficiently large probability when the number of secondary users is close to the number of channels. It can be further improved with an improved sensing policy that exploits channel state autocorrelation by considering sensing results in the previous time slot. The improved sensing policy also attempts to reduce the chance that a channel is not sensed by any of the users.

Specifically, we classify the $M$ channels into three sets in time slot $t$, including (i) the set of channels that are detected 
to be idle, denoted by $B_{0}(t)$, (ii) the set of channels that are sensed to be busy, denoted by $B_{1}(t)$, and (iii) the set of channels that are not identified due to lack of time or not being sensed, denoted by $B_{2}(t)$. Let $\left|B_{0}(t)\right|,\left|B_{1}(t)\right|$ and $\left|B_{2}(t)\right|$ be the cardinalities of $B_{0}(t), B_{1}(t)$, and $B_{2}(t)$, respectively.

If channel $m$ is in set $B_{0}(t)$ and the stop time on channel $m$ is less than the maximum stop time $\bar{K}$, one user among those $u_{m}$ users that are sensing this channel will be randomly chosen to switch to sense another channel from the set $m \cup B_{1}(t) \cup$ $B_{2}(t)$ in the $(t+1)$ th time slot. If channel $m$ is in set $B_{1}(t)$ and the stop time on channel $m$ is less than the maximum stop time $\bar{K}$, the secondary users that are sensing this channel will randomly choose a channel in $m \cup B_{2}(t)$ to sense in the $(t+1)$ th time slot. With the above approach, we can reduce the chance that a licensed channel is not sensed by any of the users. This approach also achieves the load balancing effect since it attempts to spread secondary users to the channels.

\section{B. Transmission Phase}

We adopt the $p$-persistent CSMA protocol for data channel access for secondary users during the data transmission phase. Under this protocol, a secondary user waits until channel becomes idle. Once the channel is detected idle, the secondary user attempts transmission with probability $p$. We assume the heavy load domain where each secondary user always has data to send. Therefore, once the estimate of channel $m$ exceeds the threshold $\Theta_{1}$, all the secondary users sensing the same channel will send RTS packets on the control channel with probability $p$ to contend for the transmission opportunity.

We define $P_{m}^{\text {idle }}, P_{m}^{s u c c}$ and $P_{m}^{\text {coll }}$ as the probability that there is no transmission on channel $m$, the probability that one secondary user successfully transmit a packet on channel $m$, and the probability that there is collision on channel $m$, respectively. With $p$-persistent CSMA, it follows that

$$
\begin{aligned}
P_{m}^{\text {idle }}\left(U_{m}\right) & =(1-p)^{U_{m}} \\
P_{m}^{\text {succ }}\left(U_{m}\right) & =u \times p \times(1-p)^{U_{m}-1} \\
P_{m}^{\text {coll }}\left(U_{m}\right) & =1-P_{m}^{\text {idle }}\left(U_{m}\right)-P_{m}^{\text {succ }}\left(U_{m}\right) \\
& =1-(1-p)^{U_{m}}-u \times p \times(1-p)^{U_{m}-1}
\end{aligned}
$$

Recall that $U_{m}$ is the number of secondary users that choose channel $m$ to sense and access if it is found idle.

\section{Interference Analysis}

The main challenge in designing a CR network MAC protocol is how to balance the tension between maximizing spectrum utilization for CR users and protecting primary users from harmful collisions. Let $\gamma_{m} \in(0,1)$ be the maximum tolerable collision probability to primary users on channel $\mathrm{m}$. The probability of collision caused by the secondary users should be kept below $\gamma_{m}$.

We first derive the conditional probability that channel $m$ is miss detected to be idle by $u$ secondary users after the $k$ th mini-slot as follows.

$$
\begin{aligned}
= & \sum_{D_{m}^{k} \in \Psi_{m, k u}^{1}} \sum_{D_{m}^{k-1} \in \Psi_{m,(k-1) u}^{2}} \cdots \sum_{d_{m}^{1} \in \Psi_{m, u}^{2}} \\
& \left(\begin{array}{r}
u \\
d_{m}^{1}
\end{array}\right)\left(\begin{array}{r}
u \\
d_{m}^{2}
\end{array}\right) \cdots\left(\begin{array}{c}
u \\
d_{m}^{k}
\end{array}\right)\left(\delta_{m}\right)^{D_{m}^{k}}\left(1-\delta_{m}\right)^{k u-D_{m}^{k}}
\end{aligned}
$$

Then the probability that secondary users collide with primary users on channel $m$ is

$$
\begin{array}{r}
P_{m}^{\text {intf }}=\sum_{k=1}^{\bar{K}} \sum_{u=0}^{N} \operatorname{Pr}\left(K_{m}=k \mid U_{m}=u, S_{m}=1\right) \times \\
\operatorname{Pr}\left(U_{m}=u\right) \times P_{m}^{\text {succ }}(u) .
\end{array}
$$

For primary user protection, the probability of causing collision with primary users on channel $m$ should be kept lower than or equal to $\gamma_{m}$, i.e.,

$$
P_{m}^{i n t f} \leq \gamma_{m}
$$

This constraint is used to set the channel access probability $p$ for the $p$-persistent CSMA protocol.

\section{Throughput Analysis}

Based on previous analysis, the achievable throughput of the proposed MAC protocol adopting the two sensing policies can be derived after the system attains the stationary state. Without loss of generality, we ignore the time spent on the RTS/CTS exchange, which can be approximated by a fixed amount of overhead.

The throughput of channel $m$ that is being sensed by $u$ users, denoted by $\Lambda_{m}(u)$, can be derived as

$$
\begin{aligned}
\Lambda_{m}(u)=\sum_{k=1}^{\bar{K}} \operatorname{Pr}\left(K_{m}=k \mid U_{m}=u, S_{m}=0\right) \times \\
P_{m}^{\text {succ }}(u) \times R_{m} \times \frac{(\bar{K}-k) T_{m s}+T_{\text {data }}}{T_{s}},
\end{aligned}
$$

where $R_{m}$ is the data rate of licensed channel $m, P_{m}^{\text {succ }}(u)$ is given in (15), and $T_{s}$ is the time slot duration given in (7).

Let $\vec{U}=\left[U_{1}, U_{2}, \cdots, U_{M}\right]$ be the secondary user sensing state vector, where each element $U_{m}$ is the number of secondary users that choose channel $m$ to sense and access. The aggregate throughput for the CR network, denoted by $\Lambda$, is

$$
\Lambda=\sum_{\vec{U}} \operatorname{Pr}(\vec{U}) \sum_{\vec{S}} \operatorname{Pr}(\vec{S}) \sum_{m=1}^{M} I_{\left[S_{m}=0\right]} \Lambda_{m}\left(U_{m}\right),
$$

where $\vec{S}$ is the channel state vector defined in Section II and $I_{\left[S_{m}=0\right]}$ is an indicator that channel $m$ is idle, i.e.,

$$
I_{\left[S_{m}=0\right]}= \begin{cases}1, & \text { if } S_{m}=0 \\ 0, & \text { otherwise. }\end{cases}
$$


TABLE I

SIMULATION PARAMETERS

\begin{tabular}{l|l|l}
\hline \hline Symbol & Value & Definition \\
\hline$T_{m s}$ & $9 \mu \mathrm{s}$ & mini-slot interval \\
$T_{s}$ & $1.89 \mathrm{~ms}$ & time slot interval \\
$M$ & 5 & number of licensed channels \\
$N$ & 8 & number of secondary users \\
$\eta$ & 0.3 & utilization of the licensed channels \\
$\epsilon$ & 0.3 & probability of false alarm \\
$\delta$ & 0.3 & probability of miss detection \\
$R$ & $1 \mathrm{Mb} / \mathrm{s}$ & data rate of each licensed channel \\
$\Theta_{1}$ & 0.8 & upper threshold for channel sensing \\
$\Theta_{0}$ & 0.2 & lower threshold for channel sensing \\
$\bar{K}$ & 5 & maximum stop time for channel sensing \\
\hline \hline
\end{tabular}

\section{Simulation Results}

We evaluate the performance of the proposed CR MAC protocol using a customized simulator developed with MATLAB. We compare the following four schemes in the simulations:

- a simple random sensing scheme that each user chooses one channel to sense with equal probability,

- the negotiate sensing scheme presented in [5],

- the memoryless sensing scheme as described in Section III-A1, and

- the improved sensing scheme presented in Section III-A2.

We choose the negotiate sensing scheme since it adopts a similar network model and assumptions. Each point in the plots shown in this section are the average of 10 simulation runs with different random seeds. We plot $95 \%$ confidence intervals for the simulation results, which are generally negligible in all the figures. The simulation parameters are summarized in Table I, which follow the typical values used in [5].

We first verify our throughput analysis presented in Section III. In Figs. 2, 3, and 4, we plot the throughputs for the CR MACs incorporating the memoryless sensing policy and the improved sensing policy, with both simulation and analysis curves (dashed curves). We observe that the simulation and analysis curves for the memoryless sensing CR MAC overlap completely with each other, indicating that our analysis is exact. Furthermore, although there is a gap between the simulation and analysis curves for the CR MAC with the improved sensing policy, the gap is generally very small and negligible. The gap is actually due to an approximation we used for the secondary user sensing state vector $\vec{U}$, for which deriving the exact form is non-trivial. In the analysis, we assume that the probability is 0 that a channel is not sensed by any secondary user. We find the analysis can serve as a tight upper bound for the CR MAC throughput performance when the improved sensing policy is incorporated.

In Fig. 2, we plot the throughput of the four schemes under different channel utilization values ranging from 0.3 to 0.7. As utilization of the licensed channels is increased, the transmission opportunities for secondary users are clearly reduced. Therefore the four curves are all decreasing function of $\eta$. The improved policy achieves the best performance among the four schemes, while random sensing has the poorest performance. When the channel utilization is $\eta=0.3$, the

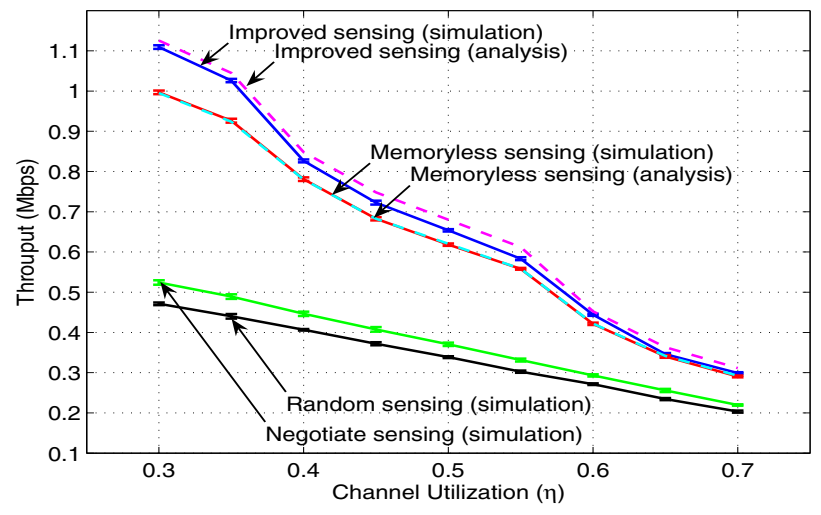

Fig. 2. Throughput versus channel utilization (with $95 \%$ confidence intervals for the simulation results).

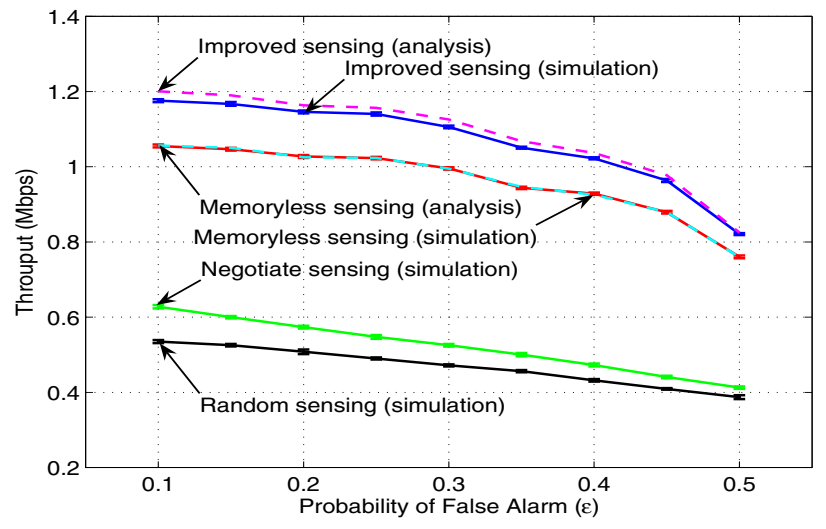

Fig. 3. Throughput versus false alarm probability (with $95 \%$ confidence intervals for the simulation results).

improved policy achieves a $10 \%$ gain in throughput over the memoryless sensing policy.

We next investigate the impact of sensing errors on the CR MAC performance. We assume identical false alarm probabilities $\epsilon_{m}=\epsilon$, and identical miss detection probabilities $\delta_{m}=\delta$ for all the licensed channels. In Fig. 3, we plot the throughputs obtained by the four schemes versus the false alarm probability $\epsilon$. Specifically, we fix $\delta$ at 0.3 and increase $\epsilon$ from 0.1 to 0.5 . Intuitively, a higher false alarm probability results in lower probability for secondary users to exploit the transmission opportunities in the licensed channels. This is illustrated in the figure, as all the four throughput curves decrease as $\epsilon$ is increased. Again, the improved sensing MAC achieves the best performance, with about $10 \%$ gain over the memoryless sensing MAC and about $200 \%$ gain over the two existing approaches.

In Fig. 4, we examine the impact of miss detection probability $\delta$ on the CR network throughput. In these simulations, we fix $\epsilon$ at 0.3 and increase $\delta$ from 0.1 to 0.5 . We find that the miss detection error has negligible impact on the throughputs of the random sensing and negotiate sensing protocols, since miss detection errors are not considered in the design of these protocols. However, both our proposed CR MAC schemes 


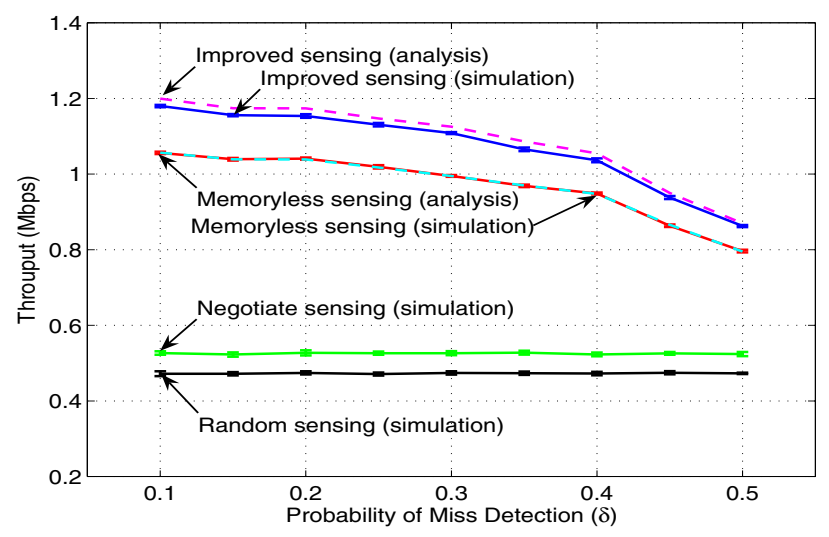

Fig. 4. Throughput versus miss detection probability (with $95 \%$ confidence intervals for the simulation results).

achieve considerable throughput gains over the random sensing and negotiate sensing schemes.

\section{RELATED WORK}

Many CR research has considered CR as "spectrum agile radio" that enables dynamic spectrum access to exploit transmission opportunities in licensed spectrum bands [1], [2]. Several MAC protocols for CR networks have been proposed in the literature. In [8], Le and Hossain propose a MAC protocol for opportunistic spectrum access in CR networks. Two channel selection schemes are proposed: uniform channel selection and spectrum opportunity-based channel selection. The latter considers the probability of spectrum availability and selects each channel with different probabilities based on the estimation of spectrum availability.

A decentralized cognitive MAC protocol is developed in [9] that allows secondary users to explore spectrum opportunities without a central coordinator or a dedicated control channel. However, the implementation is complicated and hardware demanding. This is because each secondary user needs to be equipped with multiple sensors to detect the availability of each licensed channel.

In a recent work [5], Su and Zhang propose a negotiationbased sensing policy (NSP) in which a secondary user knows which channels are already sensed and will choose a different channel to sense. In [10], the authors consider two types of hardware constraints: sensing constraint and transmission constraint. In [11], based on the information obtained by a delegate secondary user, each secondary user group selects and switches to the best data channel for data communication during the next period. In [12], Hsu, Weit and Kuo describe a policy such that a secondary user selects the channel that has the highest successful transmission probability to access. Many prior works [3], [5], [8], [10] assume perfect channel sensing, within which secondary users can always sense the channel correctly, and sensing errors are not considered. The joint design of opportunistic spectrum access and sensing policies is studied in a recent work [13] in the presence of sensing errors. The authors develop a separation principle that decouples the designs of sensing and access policy. This interesting study is based on a constrained partially observable Markov decision process (POMDP) formulation and thus has an exponentially growing computational complexity [13].

\section{CONCLUSION}

We studied the problem of MAC protocol design for CR networks in this paper. We proposed and analyzed two opportunistic multi-channel MAC protocols, adopting a memoryless sensing policy and an improved sensing policy, respectively. The impact of imperfect sensing (in the form of miss detection and false alarm) are explicitly considered in the CR MAC design. We developed analytical models to evaluate the performance of the proposed protocols. Our simulation study demonstrates the accuracy of the analysis, as well as the superior throughput performance of the proposed CR MACs over existing approaches.

\section{ACKNOWLEDGMENT}

This work is supported in part by the US National Science Foundation (NSF) under Grant ECCS-0802113 and through the Wireless Internet Center for Advanced Technology (WICAT) at Auburn University. Any opinions, findings, and conclusions or recommendations expressed in this material are those of the author(s) and do not necessarily reflect the views of the Foundation.

\section{REFERENCES}

[1] Q. Zhao and B. Sadler, "A survey of dynamic spectrum access," IEEE Signal Process. Mag., vol. 24, no. 3, pp. 79-89, May 2007.

[2] Y. Zhao, S. Mao, J. Neel, and J. Reed, "Performance evaluation of cognitive radios: metrics, utility functions, and methodologies," IEEE Proc. IEEE, vol. 97, no. 4, Apr. 2009.

[3] A. Motamedi and A. Bahai, "MAC protocol design for spectrumagile wireless networks: Stochastic control approach," in Proc. IEEE DySPAN'07, Dublin, Ireland, Apr. 2007, pp. 448-451.

[4] S. Geirhofer, L. Tong, and B. Sadler, "Cognitive medium access: Constraining interference based on experimental models," IEEE J. Sel. Areas Commun., vol. 26, no. 1, pp. 95-105, Jan. 2008.

[5] H. Su and X. Zhang, "Cross-layer based opportunistic MAC protocols for QoS provisionings over cognitive radio wireless networks," IEEE $J$. Sel. Areas Commun., vol. 26, no. 1, pp. 118-129, Jan. 2008.

[6] D. Hu, S. Mao, and J. Reed, "On video multicast in cognitive radio networks," in Proc. IEEE INFOCOM'09, Rio de Janeiro, Brazil, Apr. 2009.

[7] H. Nan, T.-I. Hyon, and S.-J. Yoo, "Distributed coordinated spectrum sharing MAC protocol for cognitive radio," in Proc. IEEE DySPAN'07, Dublin, Ireland, Apr. 2007, pp. 240-249.

[8] L. Le and E. Hossain, "OSA-MAC: A MAC protocol for opportunistic spectrum access in cognitive radio networks," in Proc. IEEE WCNC'08, Las Vegas, NV, Mar./Apr. 2008, pp. 1426-1430.

[9] Q. Zhao, L. Tong, A. Swami, and Y. Chen, "Decentralized cognitive MAC for opportunistic spectrum access in ad hoc networks: A POMDP framework," IEEE J. Sel. Areas Commun., vol. 25, no. 3, pp. 589-600, Apr. 2007.

[10] J. Jia, Q. Zhang, and X. Shen, "HC-MAC: A hardware-constrained cognitive MAC for efficient spectrum management," IEEE J. Sel. Areas Commun., vol. 26, no. 1, pp. 106-117, Jan. 2008.

[11] B. Hamdaoui and K. Shin, "OS-MAC: An efficient MAC protocol for spectrum-agile wireless networks," IEEE Trans. Mobile Comput., vol. 7, no. 8, pp. 915-930, Aug. 2008.

[12] A.-C. Hsu, D. Weit, and C.-C. Kuo, "A cognitive MAC protocol using statistical channel allocation for wireless ad-hoc networks," in Proc. IEEE WCNC'07, Hong Kong, China, Mar. 2007, pp. 105-110.

[13] Y. Chen, Q. Zhao, and A. Swami, "Joint design and separation principle for opportunistic spectrum access in the presence of sensing errors," IEEE Trans. Inf. Theory, vol. 54, no. 5, pp. 2053-2071, May 2008. 